\title{
Long noncoding RNA LINCOI 296 promotes cancer-cell proliferation and metastasis in urothelial carcinoma of the bladder
}

This article was published in the following Dove Medical Press journal:

OncoTargets and Therapy

\section{Xiaofei Wang' \\ Lei Wang' \\ Yanbing Gong ${ }^{2}$ \\ Zhenzhen Liu $^{3}$ \\ Yingchao Qin ${ }^{4}$ \\ Jia Chen' \\ Ningcheng $\mathrm{Li}^{\prime}$}

'Department of Urology, Peking University Shougang Hospital,

Beijing I00I44, People's Republic of China; ${ }^{2}$ Department of Science Research, Peking University Shougang Hospital, Beijing I00I44, People's Republic of China; ${ }^{3}$ Key Laboratory of Carcinogenesis and Translational Research (Ministry of Education), Hepatopancreatobiliary Surgery Department I, Peking University Cancer Hospital and Institute, Beijing I00 I42, People's Republic of China; ${ }^{4}$ Department of General Surgery, Chaoyang District Shuangqiao Hospital, Beijing 100024, People's Republic of China
Correspondence: Ningcheng Li Department of Urology, Peking University Shougang Hospital, 9 Jinyuanzhuang Road, Shijingshan District, Beijing 100I44, People's Republic of China Tel +86 I39 I070 835I

Email ningchenli@I26.com
Purpose: Long noncoding RNAs (lncRNAs) play an important role in the tumorigenesis and progression of human cancer. This research was performed to investigate the role of LINC01296 in clinical characteristics, biological functions and molecular mechanisms of bladder cancer.

Materials and methods: In this study, expressions of LINC01296 in cancer tissues and normal tissues were firstly compared using the Gene Expression Profiling Interactive Analysis database. Subsequently, a microarray data analysis was performed to compare lncRNA and mRNA expression profiles in four pairs of human bladder cancer samples. Then, quantitative real-time polymerase chain reaction (qRT-PCR) was used to detect the expression of LINC01296 in bladder cancer tissues. The association between LINC01296 expressions and clinicopathological characteristics of bladder cancer was analyzed by Kaplan-Meier analysis and the Cox proportional-hazard model. The biological functions and molecular mechanisms of LINC01296 in bladder cancer were studied by MTT assay, colony-formation assay, cell cycle analysis, transwell migration assay, wound healing assay, qRT-PCR analysis and Western blot assay.

Results: The expression of LINC01296 was significantly higher in most cancer tissues than that in adjacent normal tissues, and was positively correlated with clinical stages of the cancer ( $P=0.016)$, lymph node metastasis $(P=0.034)$, and pathologic grades $(P=0.012)$. The increased level of LINC01296 was associated with a poorer prognosis and shorter survival of the patients. Multivariate analysis showed that the LINC01296 expression was an independent predictor of overall survival in bladder cancer. Additionally, LINC01296 knockdown inhibited the proliferation, migration and progression of cell cycle of bladder cancer cells, and was involved in the regulation of epithelial-mesenchymal transition.

Conclusion: The findings of this study suggested that LINC01296 promotes progression of bladder cancer, and potentially acts as a biomarker and therapeutic target of bladder cancer.

Keywords: bladder cancer, long noncoding RNA, biological function, biomarker, therapeutic target

\section{Introduction}

Bladder cancer is one of the most common malignancies worldwide. The incidence of bladder cancer ranks eleventh among all malignant tumors and sixth among malignant tumors in men. ${ }^{1}$ The latest data from global cancer statistics indicate that the annual new cases and deaths of bladder cancer are up to 440,000 and 150,000, respectively. Bladder cancer is associated with different histopathological types and clinical manifestations, which make it more difficult to diagnose and treat. ${ }^{2,3}$ The most common histopathological type of bladder cancer is urinary epithelial carcinoma. In addition, the 5-year survival rate of patients with nonmuscular invasive bladder cancer is up 
to $85 \%$, while the 5 -year survival rate of patients with muscular invasive bladder cancer is only $6 \%$ due to frequent and distant metastasis. ${ }^{4-7}$ The mortality of patients with invasive and metastatic bladder cancer remains high despite treatments with surgery, radiotherapy, and chemotherapy. ${ }^{8}$

Less than $2 \%$ of the human genome contains protein encoding genes, while the vast majority of the human genome (98\%) consists of nonprotein-coding RNAs (ncRNAs) that do not encode any protein. ${ }^{9}$ In recent years, ncRNAs, including small ncRNAs (eg, microRNAs) and long noncoding RNAs (lncRNAs), have been shown to play a key role in various biological processes, particularly in cancer. ${ }^{10-16}$ LncRNAs are a type of ncRNAs longer than 200 nucleotides whose RNA polymerase II transcripts do not contain an open reading frame. ${ }^{17}$ LncRNAs were originally thought to be the "noise" of genome transcription with no biological functions. However, an increasing number of studies have revealed that lncRNAs take part in regulating gene expression at transcriptional, post-transcriptional, and epigenetic levels by interacting with DNA, RNA, and protein. The biological functions of lncRNAs include chromosome silencing, genomic imprinting, chromatin modification, transcriptional activation, transcriptional interference, and nuclear transport. ${ }^{18}$ LncRNAs have been demonstrated to play important roles in the development and progression of bladder cancer, although such roles of lncRNA in bladder cancer development remain unclear and require further investigation. LINC01296, a novel intergenic lncRNA located at chromosome 14q11.2, was previously discovered by microarray analysis. In this research, it was demonstrated that lncRNA LINC01296 was aberrantly expressed in bladder cancer tissues and positively associated with clinical stages of bladder cancer, lymph node metastasis and pathologic grades of bladder cancer. Furthermore, the functional analysis revealed that LINC01296 could promote the proliferation and metastasis of cancer cells in urothelial carcinoma of bladder. Moreover, LINC01296 could promote tumor development and progression through epithelialmesenchymal transition (EMT).

\section{Materials and methods \\ Ethics statement}

Written informed consent was obtained from all patients. The protocol was approved by the Ethics Committee of Peking University Shougang Hospital (Beijing, People's Republic of China).

\section{Patient and tissue samples}

A total of 78 cases of specimens, including 54 samples of bladder cancer tissues and 24 samples of adjacent normal bladder tissues, were obtained from Peking University Shougang Hospital between July 2014 and July 2017 from patients who had undergone surgical resection of bladder cancer. The patients were diagnosed as having bladder cancer based on the histopathological evaluation. All collected tissue samples were immediately snap frozen in liquid nitrogen after resection and stored at $-196^{\circ} \mathrm{C}$ for subsequent use. The methods used in this research followed the requirements of relevant guidelines and regulations.

\section{Cell culture}

The human bladder cancer cell lines (RT4, T24, and 5637) were obtained from the central laboratories of Peking University Third Hospital and Peking University First Hospital. These cell lines were purchased from the Institute of Biochemistry and Cell Biology of the Chinese Academy of Sciences (Shanghai, People's Republic of China). At $37^{\circ} \mathrm{C}$ in a humidified air atmosphere containing $5 \% \mathrm{CO}_{2}$, all cell lines were grown and maintained in RPMI-1640 medium or DMEM Medium (Gibco ${ }^{\circledR}$; Thermo Fisher Scientific, Waltham, MA, USA) supplemented with $10 \%$ fetal bovine serum (Thermo Fisher Scientific), $100 \mathrm{U} / \mathrm{mL}$ penicillin sodium, and $100 \mathrm{mg} / \mathrm{mL}$ streptomycin.

\section{Total RNA isolation and quantitative real-time polymerase chain reaction (qRT-PCR)}

Total RNA was extracted from tissues and cultured cells using a TRIzol reagent (Thermo Fisher Scientific) according to the manufacturer's protocols. For reverse-transcription polymerase chain reaction (PCR), the reaction was carried out in a final volume of $10 \mu \mathrm{L}$ containing $1 \mu \mathrm{g}$ total RNA, $7 \mu \mathrm{L}$ nuclease-free water, $2 \mu \mathrm{L} 5 \times \mathrm{RT}$ Buffer, $0.5 \mu \mathrm{L}$ RT Enzyme Mix, and $0.5 \mu \mathrm{L}$ Random Primer Mix, by using a Rever Tra Ace qPCR RT Kit (Toyobo Co. Ltd, Osaka, Japan). Reverse transcription was performed at $37^{\circ} \mathrm{C}$ for 60 minutes, followed by $98^{\circ} \mathrm{C}$ for 5 minutes and ice bath for 15 seconds according to the manufacturer's instructions. Quantitative real-time PCR (qRT-PCR) was performed on an ABI 7500 (Thermo Fisher Scientific) instrument using a KAPA SYBR Green FAST qPCR Kit (KAPA, Wilmington, MA, US) according to the manufacturer's instructions. The reaction was performed in a $20 \mu \mathrm{L}$ reaction volume containing $10 \mu \mathrm{L}$ of KAPA SYBR ${ }^{\circledR}$ FAST qPCR Master Mix (2X) Universal, $0.4 \mu \mathrm{L}$ of PCR forward primer $(10 \mu \mathrm{M}), 0.4 \mu \mathrm{L}$ of PCR reverse primer $(10 \mu \mathrm{M}), 0.4 \mu \mathrm{L}$ of ROX low, $8.8 \mu \mathrm{L}$ of double-distilled water, and less than $2 \mu \mathrm{L}$ of template DNA. The qRT-PCR protocol included an initial denaturation step 
$\left(95^{\circ} \mathrm{C}\right.$ for 3 minutes) and 40 cycles of denaturation $\left(95^{\circ} \mathrm{C}\right.$ for 15 seconds), annealing $\left(58^{\circ} \mathrm{C}\right.$ for 30 seconds $)$ and extension ( $72^{\circ} \mathrm{C}$ for 1 minute). GAPDH was used as the internal control. The qRT-PCR values were normalized to GAPDH and calculated using the $2^{-\Delta \Delta \mathrm{CT}}$ method. In this method, $\Delta \mathrm{CT}$ is the quantitative value of the target gene (LINC01296) relative to the internal reference gene (GAPDH) in the experimental group or the control group, while $2^{-\Delta \Delta \mathrm{CT}}$ is the fold change of the expression of the target gene (LINC01296) in the experimental group when compared with that in the control group. Each experiment was performed in triplicate and repeated three times. The specific primers for PCR were as follows:

LINC01296, forward 5'-ACTCCTGGCCTCAAACA ATTATTC-3', reverse, 5'-AAGTGGGTAAGCTGAAACTGA AAC-3';

GAPDH, forward, 5'-GAACGGGAAGCTCACTGG-3', reverse, 5'-GCCTGCTTCACCACCTTCT-3'.

E-cadherin, forward, 5'-AATAGTGCCTAAAGTG CTGC-3', reverse, 5'-AGACCCACCTCAATCATCCT-3'. $\mathrm{N}$-cadherin, forward, 5'-ATCCTACTGGACGGT TCG-3', reverse, 5'-TTGGCTAATGGCACTTGA-3'.

\section{Cell transfection}

A LncRNA Smart Silencer targeting LINC01296 RNA and a scramble control were synthesized by Guangzhou RiboBio Co., Ltd (Guangzhou, People's Republic of China). The inhibitor and the control RNA were then transfected into bladder cancer cell lines using Lipofectamine 2000 (Thermo Fisher Scientific) according to the manufacturer's instructions. In brief, the cells were cultured in six-well plates until their density reached $70 \%$ prior to transfection. After the transfection, the cells were harvested at 24 hours, 48 hours, and 72 hours for qRT-PCR analysis. The RiboTM lncRNA Smart Silencer for human LINC01296 was a pool containing three siRNAs and three antisense oligonucleotides:

siRNA-1 target sequence: GCACACTGTTTCAA CTCTC

siRNA-2 target sequence: GCACTTCACTGTCA CACTC

siRNA-3 target sequence: GCAGCTGGGATTTG TAAGT

antisense oligonucleotides target sequence-1:

CCATAGGTCACCAGCTTCAC

antisense oligonucleotides target sequence-2:

GCTCAGCTTCCACACTCAGA antisense oligonucleotides target sequence-3:

GGAGCTCAAAGGATGCATTT.

\section{Cell proliferation assay}

Cell proliferation was measured using a 3-(4,5dimethylthiazole-2-yl)-2,5-diphenyltetrazolium bromide (MTT) assay (Roche Applied Science, Mannheim, Germany). The bladder cancer cells (T24 and 5637, 2,000 cells per well) transfected with IncRNA inhibitors were seeded into 96-well plates, with each well containing 200 $\mu \mathrm{L}$ of a complete culture medium. Cell viability was tested every 24 hours following the manufacturer's protocol. The assay was repeatedly performed in triplicate.

\section{Colony-formation assay}

For the colony formation assay, a total of 1,000 cells/well were seeded into a six-well plate and maintained in a medium containing $10 \%$ FBS. The medium was replaced every 4 days. After being cultured for 2 weeks, the medium was removed and the cells were washed twice with phosphate buffered saline (PBS), and then the colonies were fixed with $4 \%$ paraformaldehyde for 10 minutes and stained with $0.1 \%$ crystal violet for 30 minutes. Finally, visible colonies were examined and counted under a microscope. Each treatment group was assessed in triplicate.

\section{Cell cycle analysis}

T24 and 5637 cells transfected with lncRNA inhibitors were harvested at 48 hours after transfection. The cells were washed with PBS and fixed in ethanol at $4^{\circ} \mathrm{C}$ overnight. The cells were then stained with PI using the CycleTESTTM PLUS DNA Reagent Kit (BD Biosciences, San Jose, CA, USA) according to the protocol provided by the manufacturer. Subsequently, the stained cells were analyzed by FACScan. The percentages of the cells in G0-G1, S, and G2-M phases were calculated and compared among different groups.

\section{Transwell migration assay}

Cell migration assays were performed in transwell chambers (8- $\mu \mathrm{m}$ pore size; EMD Millipore, Billerica, MA, USA). In brief, the cells $\left(4 \times 10^{4}\right)$ were collected at 48 hours after transfection, resuspended in $200 \mu \mathrm{L}$ of serum-free medium and seeded in the upper chamber of a transwell, while a culture medium $(900 \mu \mathrm{L})$ containing $10 \%$ fetal bovine serum was added to the lower chamber. After 24 hours of incubation at $37^{\circ} \mathrm{C}$ in a humidified atmosphere containing $5 \% \mathrm{CO}_{2}$, the cells remaining on the upper membrane of the transwell chambers were removed with a piece of cotton wool, while the cells that have migrated through the membrane were fixed 
with polyoxymethylene for 10 minutes and stained with $0.1 \%$ crystal violet for 30 minutes before counting. Experiments were independently repeated three times.

\section{Wound healing assay}

Bladder cancer cells transfected with lncRNA inhibitors were collected after 48 hours of transfection and seeded into six-well plates $\left(1 \times 10^{5}\right.$ cells per well). At $90 \%-95 \%$ confluence, the culture solution was discarded and the monolayer of the cells was wounded by manually scraping with a $200 \mu \mathrm{L}$ sterile pipette tip. The cell monolayer was then washed with PBS and cultured under standard conditions. At 0 hour, 18 hours, and 24 hours after the wound was generated, the images of the cell monolayer were taken using a TH4-200 inverted microscope (Olympus Corporation, Tokyo, Japan), and the migration rate was quantified using the distance among the cells.

\section{Western blot analysis}

T24 and 5637 cells transfected with lncRNA inhibitors for 48 hours were washed twice with PBS before their total protein was extracted using a RIPA buffer according to instructions. The protein concentration was determined using a BCA protein assay kit (Thermo Fisher Scientific), and bovine serum albumin was used as the standard for the assay. Sixty micrograms of protein was separated by $8 \%$ SDS-polyacrylamide gel electrophoresis (SDS-PAGE) and transferred onto $0.4-\mu \mathrm{m}$ nitrocellulose membranes (Sigma Aldrich, St Louis, MO, USA), and then incubated with specific primary antibodies. $\beta$-actin antibody was used as control. Primary anti- $\beta$-actin, anti-E-cadherin, and anti$\mathrm{N}$-cadherin antibodies were purchased from Santa Cruz Biotechnology Inc. (Dallas, TX, USA).

\section{Statistical analyses}

Data from at least three independent experiments were presented as the mean \pm SD. All statistical analyses were carried out using SPSS 20.0 (IBM Corporation, Armonk, NY, USA) or GraphPad Prism five software (GraphPad Software Inc., La Jolla, CA, USA). The significance of difference between two/multiple groups was evaluated using Student's $t$-tests or one-way ANOVA. Pearson's chi-squared test was used for the analysis of correlation between clinicopathological features and LINC01296 expressions in bladder cancer patients. Kaplan-Meier survival analysis was used for the analysis of survival rates, while $P$-values were calculated by log-rank test. Multivariate analysis was performed to evaluate the correlation between clinical features, genetic features and overall survival (OS) using a Cox proportional hazard model. $P<0.05$ indicated significant difference.

\section{Results}

LncRNA LINC01296 was upregulated in human bladder cancer and was positively correlated with clinical stages of bladder cancer, lymph node metastasis and pathologic grades of bladder cancer.

In this study, using the Gene Expression Profiling Interactive Analysis (GEPIA) database, the relative expression of LINC01296 in human cancer tissues was compared with that in normal tissues, ${ }^{19}$ and the results showed that the LINC01296 expression was significantly upregulated in most cancer tissues, including bladder cancer tissues, compared with that in adjacent normal tissues (Figure 1A and B). Furthermore, the expression profiles of lncRNA and mRNA in four pairs of human bladder cancer samples were screened through a microarray data analysis (Figure 1C). Subsequently, samples from a total of 78 subjects, including 54 cases of bladder cancer tissues and 24 cases of adjacent normal tissues, were collected to measure the expression levels of LINC 01296 by qRT-PCR. The results showed that LINC01296 expressions were significantly upregulated in bladder cancer tissues compared with adjacent nontumor tissues (Figure 1D). To further investigate the correlation between LINC01296 expressions and clinicopathological features of bladder cancer, the above samples were divided into high (above the mean, $\mathrm{n}=25$ ) and low (below the mean, $\mathrm{n}=29$ ) LINC01296 expression groups (Figure 1E). As shown in Table 1, the increased level of LINC01296 in bladder cancer tissues was significantly correlated with advanced stages of the cancer $(P=0.016)$, lymph node metastasis $(P=0.034)$ and pathologic grades of the cancer $(P=0.012)$ (Figure 1F). However, LINC01296 expressions were not associated with other factors, including gender $(P=0.951)$ and age $(P=0.128)$, of bladder cancer patients (Table 1$)$.

\section{Relationship between LINCOI 296 expressions and prognosis of patients with bladder cancer}

The association between LINC01296 expressions and the prognosis of bladder cancer patients was evaluated by Kaplan-Meier analysis and log-rank tests. The results of Kaplan-Meier survival analysis indicated that high LINC01296 expressions were significantly associated with worse OS compared with low LINC01296 expressions in 
A

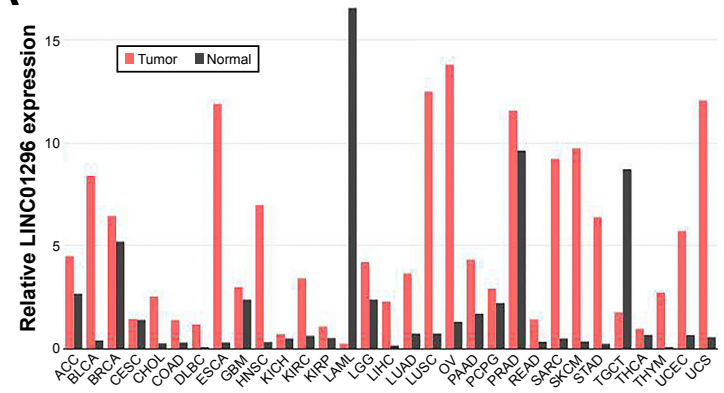

B

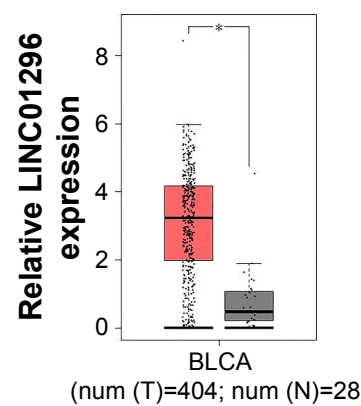

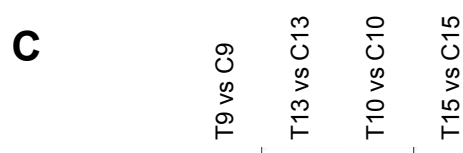

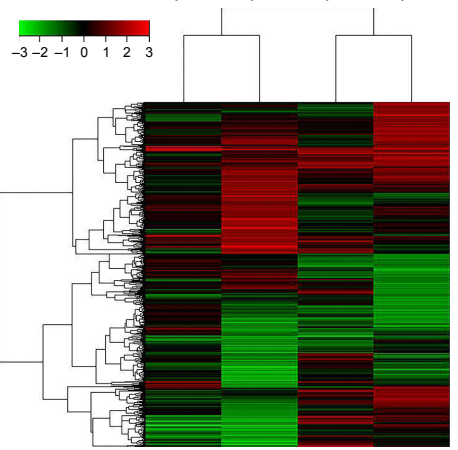

$($ num $(T)=404 ; \operatorname{num}(N)=28)$
D

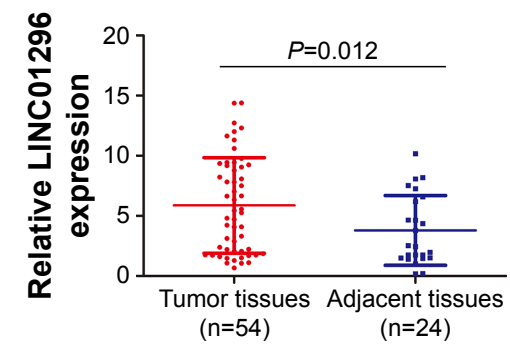

E

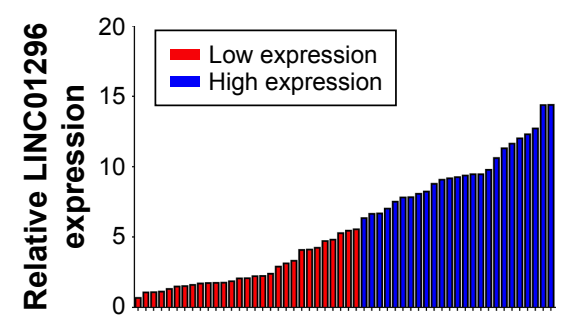

$\mathbf{F}$

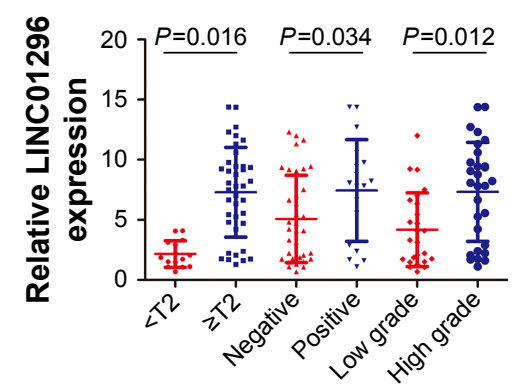

Figure I LINCOI 296 was upregulated in bladder cancer and the expression level of LINC0I296 was correlated with clinicopathological features of bladder cancer patients. Notes: (A) Comparison of relative expressions of LINC0I 296 in cancer and normal tissues using the GEPIA database. (B) Comparison of relative expressions of LINC0I296 in bladder cancer and adjacent normal tissues using the GEPIA database. (C) A microarray containing 37,000 IncRNA and 34,000 mRNA probes was used to screen differentially expressed IncRNAs in four pairs of bladder cancer patients. (D) Comparison of relative expressions of LINC0I296 in 54 bladder cancer tissues and 24 normal bladder tissues using qRT-PCR. (E) Two groups were set up according to the mean expression of LINC0I296 in tumor tissues. (F) The LINC0I296 expression was significantly higher in patients with a higher tumor stage, lymph node metastasis and a higher pathologic grade. $* P<0.05$.

Abbreviations: BLCA, bladder urothelial carcinoma; GAPDH, glyceraldehyde 3-phosphate dehydrogenase; GEPIA, Gene Expression Profiling Interactive Analysis; IncRNA, long noncoding RNA; mRNA, messenger RNA; qRT-PCR, quantitative real-time polymerase chain reaction.

bladder cancer (Figure 2), indicating that LINC01296 may play an important role in the prognosis of bladder cancer patients. Furthermore, the correlation between OS of bladder cancer patients and several other factors was evaluated by COX univariate and multivariate analyses. The results of the univariate analysis indicated that tumor stages, lymph node metastasis, pathologic grades of bladder cancer, and the relative expression of LINC01296 were significantly associated with OS of bladder cancer patients. The results of multivariate analysis showed that a higher pathologic grade and a high expression level of LINC01296 were independent risk factors for the prognosis of bladder cancer patients (Table 2).

\section{LINCOI296 expressions in bladder cancer cells}

In preliminary experiments, it was found that LINC01296 expressions were similarly upregulated in bladder cancer cell lines compared with normal bladder cell lines. ${ }^{20}$ Subsequently, LINC01296 expressions in a panel of bladder cancer cell lines, including RT4, T24 and 5637, were examined by qRT-PCR analysis. It was found that LINC01296 expressions were higher in 5637 and T24 cells than in RT4 cells (Figure 3A). Thus, 5637 and T24 cell lines were selected for subsequent experiments. Then, in order to verify the role of LINC01296 dysregulation in bladder cancer cell lines, LINC01296 expressions were knocked down in 5637 and T24 cells by short interference RNAs (siRNAs), while the qRT-PCR analysis of LINC01296 expressions was performed at 24 hours, 48 hours and 72 hours post-transfection. The results showed that, compared with that in control cells, the LINC01296 expression was most significantly reduced by 48 hours of si-LINC01296 transfection (Figure 3D and E). Therefore, various cell lines were transfected by si-LINC01296 for 48 hours in the subsequent study. 
Table I The correlation between LINC0I296 expressions and clinicopathological characteristics of bladder cancer $(n=54)$

\begin{tabular}{|l|l|l|l|l|}
\hline LINC0 I 296 expression & $\begin{array}{l}\text { Low ex- } \\
\text { pression } \\
\text { cases }\end{array}$ & $\begin{array}{l}\text { High ex- } \\
\text { pression } \\
\text { cases }\end{array}$ & $\begin{array}{l}\text { Chi-squared } \\
\text { test }\end{array}$ & P-value \\
\hline Characteristics patients & $\mathbf{n = 2 9}$ & $\mathbf{n}=\mathbf{2 5}$ & & \\
\hline Gender & & & 0.004 & 0.95 I \\
\hline Male & 16 & 14 & & \\
\hline Female & 13 & 11 & & \\
\hline Age (years) & & & 2.323 & 0.128 \\
\hline$\leq 55$ & 14 & 7 & & \\
\hline$>55$ & 15 & 18 & & 0.016 \\
\hline Tumor stage & & & 5.776 & \\
\hline$<$ T2 & 12 & 3 & & 0.034 \\
\hline$\geq$ T2 & 17 & 22 & & \\
\hline $\begin{array}{l}\text { Lymph-node } \\
\text { metastasis }\end{array}$ & & & 4.506 & \\
\hline Negative & 23 & 13 & & \\
\hline Positive & 6 & 12 & & \\
\hline $\begin{array}{l}\text { Pathologic } \\
\text { grade }\end{array}$ & & & 6.268 & \\
\hline Low grade & 18 & 7 & & \\
\hline High grade & 11 & 18 & & \\
\hline
\end{tabular}

Knockdown of LINC0I296 inhibited the proliferation of bladder cancer cells and promoted cell cycle arrest

To further investigate the biological functions of LINC01296 in bladder cancer cells, an MTT assay was performed, and the results showed that the viability of T24 and 5637 cells

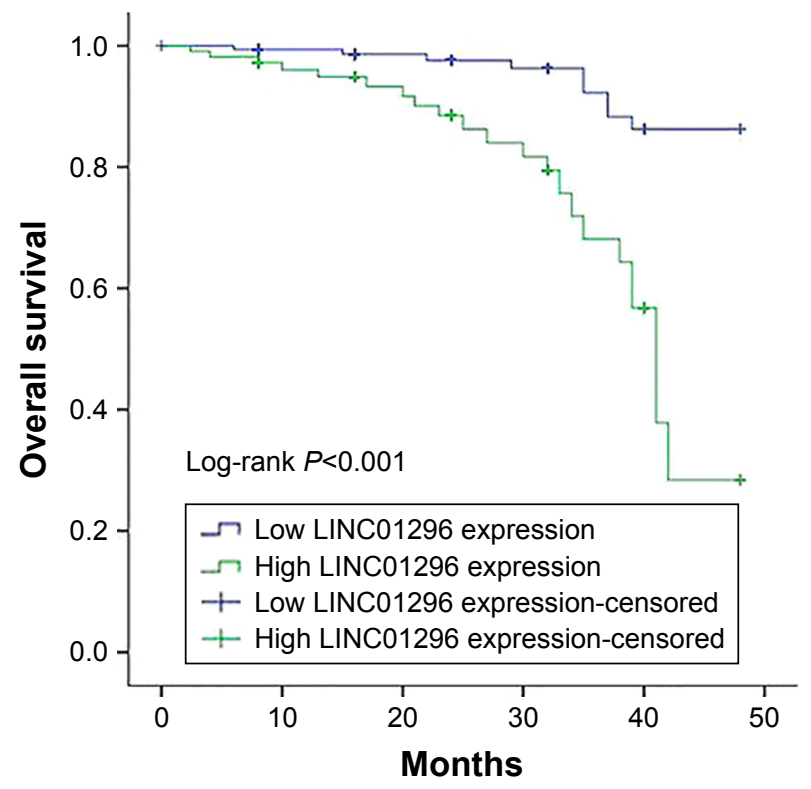

Figure 2 Kaplan-Meier survival analysis curves revealed an association between a higher expression level of LINC0I296 and a shorter overall survival $(P<0.00 \mathrm{I})$. transfected with si-LINC01296 was significantly impaired (Figure 3B and C). Similarly, the results of a colony formation assay indicated that the clonogenic survival of both T24 and 5637 cell lines was apparently decreased following the inhibition of LINC01296 (Figure 3F and G). Furthermore, to determine whether the effects of LINC01296 on the proliferation of bladder cancer cells were mediated via the effects of LINC01296 on cell cycle progression, a flow cytometry assay was performed. The results showed that the percentage of G0/G1 cells increased after bladder cancer cells were transfected with si-LINC01296, along with decreased proportions of cells in S and G2/M phases (Figure 3H and I). In a word, these data indicated that LINC01296 could promote the proliferation and cell cycle progression of bladder cancer cells.

\section{Knockdown of LINCOI296 inhibited the migration of bladder cancer cells via the regulation of EMT}

Migration and metastasis of cancer cells are an important factor of cancer progression. To further clarify the effects of LINC01296 on the metastasis of bladder tumor, wound healing assay and transwell migration assay were conducted. The results of wound healing assay and transwell assay revealed that a decreased level of LINC01296 significantly inhibited the migration of bladder cancer cells (Figure 4A-D). These data showed that the knockdown of LINC01296 exerted a tumor-suppressive effect to inhibit the migration of bladder cancer cells. Since EMT has been shown to play an important role in cancer cell metastasis, the effect of LINC01296 on EMT was investigated in this study by analyzing the expression of EMT markers after the knockdown of LINC01296. The results of qRT-PCR analysis showed that LINC011296 knockdown increased the mRNA level of E-cadherin while decreasing the mRNA level of $\mathrm{N}$-cadherin (Figure 4E). The results of Western blot assays suggested that the protein level of N-cadherin was decreased in bladder cancer cells whose LINC01296 expression was silenced (Figure 4F).

\section{Discussion}

As one of the most prevalent carcinomas worldwide, bladder cancer is extremely sensitive to chemotherapy. Although cisplatin and gemcitabine are widely used in the chemotherapy of bladder cancer, the emergence of drug resistance greatly limits their long-term effectiveness. ${ }^{21}$ Furthermore, invasive bladder tumors with the ability to attack the muscularis propria layer are more aggressive and lead to a much worse 
Table 2 Univariate and multivariate analyses on overall survival of bladder cancer patients $(n=54)$

\begin{tabular}{|c|c|c|c|c|c|c|}
\hline \multirow[t]{2}{*}{ Characteristics } & \multicolumn{3}{|c|}{ Univariate analysis } & \multicolumn{3}{|c|}{ Multivariate analysis } \\
\hline & HR & $95 \% \mathrm{Cl}$ & $P$-value & HR & $95 \% \mathrm{Cl}$ & $P$-value \\
\hline \multicolumn{7}{|l|}{ Gender } \\
\hline Male vs female & 0.900 & $0.443-1.827$ & 0.770 & & & \\
\hline \multicolumn{7}{|l|}{ Age (years) } \\
\hline$\leq 55$ vs $>55$ & 1.567 & $0.737-3.334$ & 0.243 & & & \\
\hline \multicolumn{7}{|l|}{ Tumor stage } \\
\hline$<\mathrm{T} 2$ vs $\geq \mathrm{T} 2$ & 6.241 & $|.886-20.65|$ & 0.003 & 2.330 & $0.604-8.987$ & 0.220 \\
\hline \multicolumn{7}{|l|}{ Lymph node metastasis } \\
\hline Negative vs positive & 3.007 & $1.48 \mathrm{I}-6.105$ & 0.002 & 0.879 & $0.387-2.000$ & 0.759 \\
\hline \multicolumn{7}{|l|}{ Pathologic grade } \\
\hline Low grade vs high grade & 7.079 & $2.870-17.457$ & $<0.001$ & 5.358 & $1.684-17.044$ & 0.004 \\
\hline \multicolumn{7}{|l|}{ LINC0I 296 expression } \\
\hline Low vs high & 5.742 & $2.569-12.832$ & $<0.001$ & 5.017 & $2.063-12.200$ & $<0.001$ \\
\hline
\end{tabular}

A

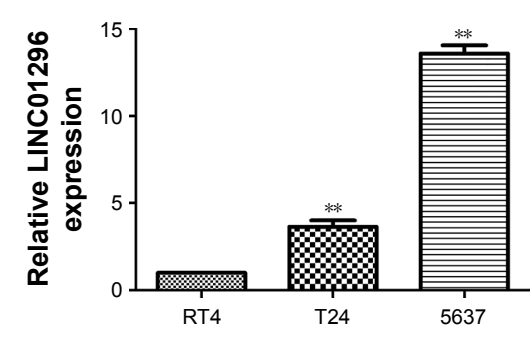

B

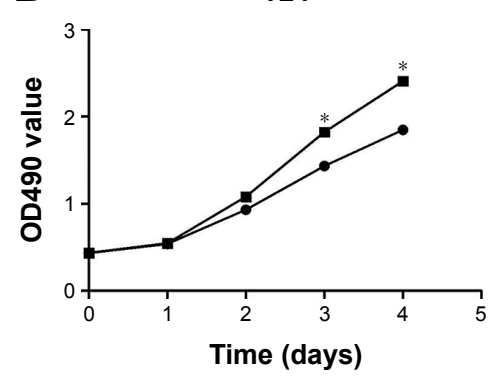

C

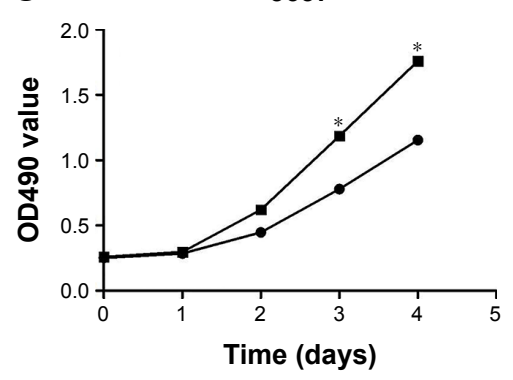

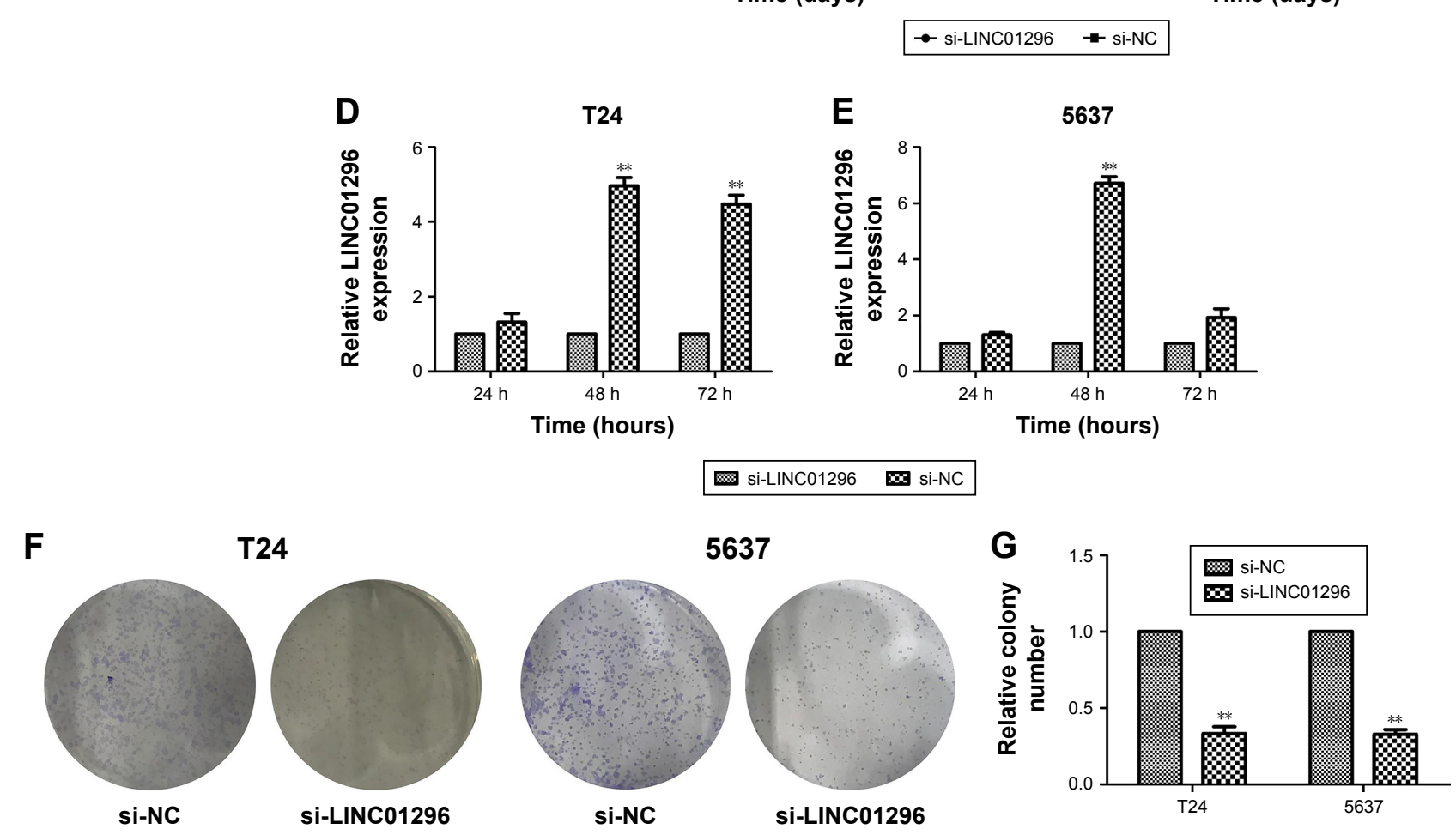

Figure 3 (Continued) 
H

T24

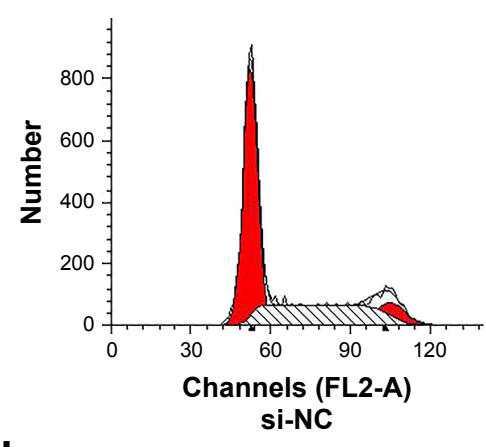

I

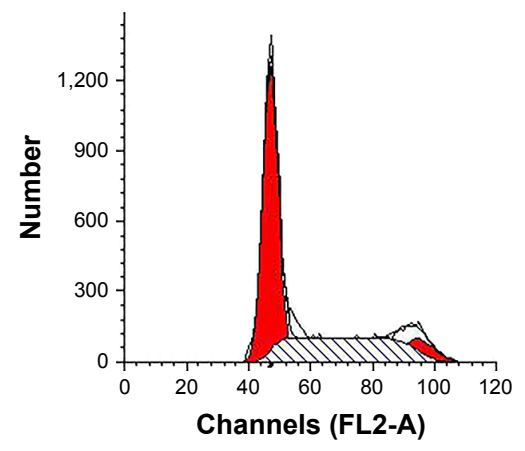

si-NC
T24

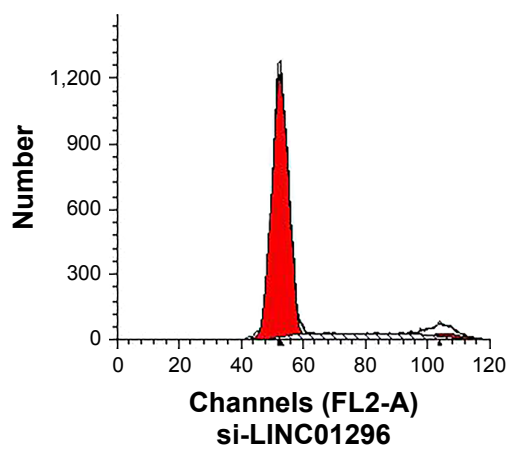

5637

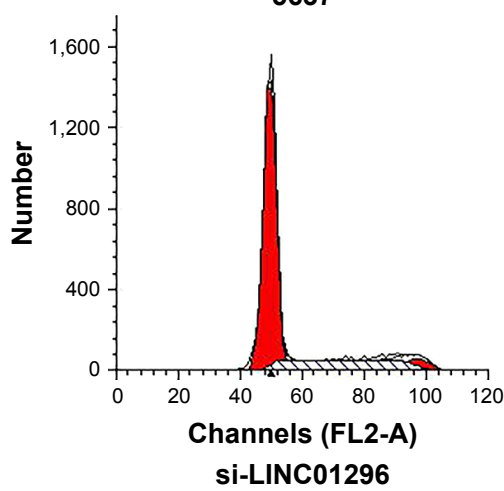

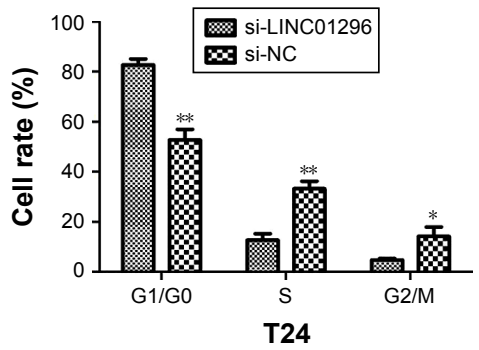

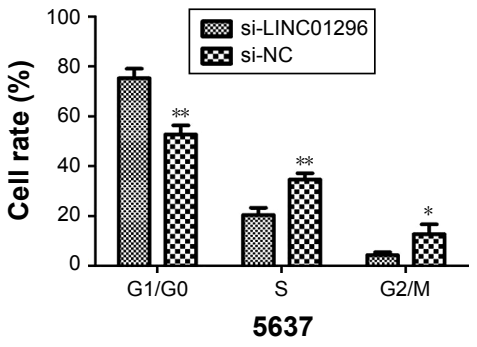

$\square$ Debris $\square$ Aggregates $\square$ Dip G1 $\square$ Dip G2 $\square$ Dip S

Figure 3 LINCOI296 knockdown inhibited the proliferation of bladder cancer cells.

Notes: (A) Relative LINC0I296 expressions in human bladder cancer cell lines (RT4, T24, and 5637). (B, C) MTT assays were performed to measure the proliferation viability of T24 and 5637 cells after transfection. (D, E) qRT-PCR results of LINC0I296 expressions following the treatment of T24 and 5637 cells with anti-LINC0I296 siRNA. (F, G) Colony formation assays were used to measure the number of clone formation of bladder cancer cells after transfection. (H, I) Flow cytometry assays were performed to analyze the cell cycle progression of T24 and 5637 cells after they were transfected with anti-LINC0I296 siRNA. Data are presented as mean \pm SD of three independent experiments. $* P<0.05$, $* * P<0.01$.

Abbreviations: NC, negative control; qRT-PCR, quantitative real-time polymerase chain reaction; siRNA, short interference RNA.

prognosis of the patients. ${ }^{22}$ The recurrence of bladder cancer, the onset of drug resistance and a high rate of cancer progression are major obstacles faced by the treatment of bladder cancer, thus highlighting the urgent need for the discovery of new biomarkers in the clinical diagnosis and therapy of bladder cancer. ${ }^{23}$ Recently, more studies have indicated that IncRNAs play an important role in tumorigenesis and development of tumors by participating in essential cellular
A
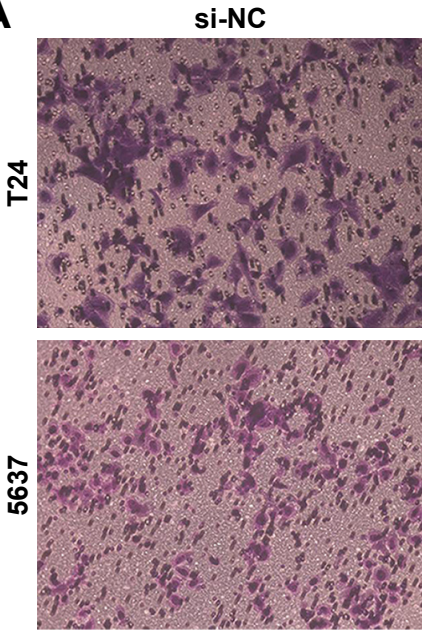

si-LINC01296
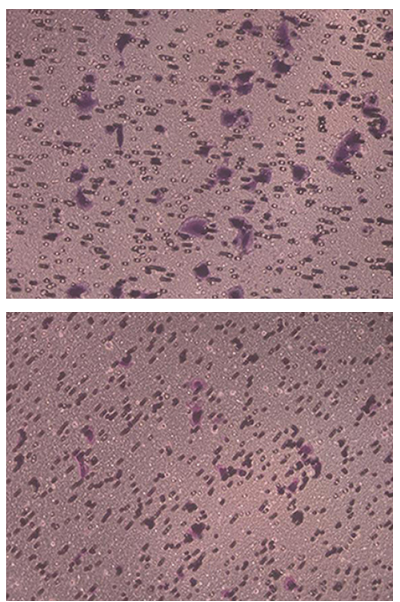

B

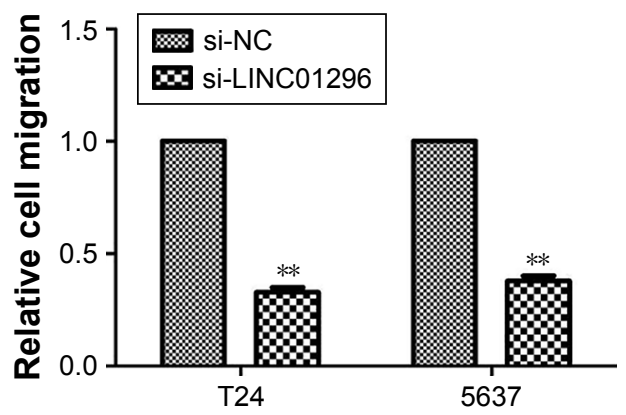

Figure 4 (Continued) 
C
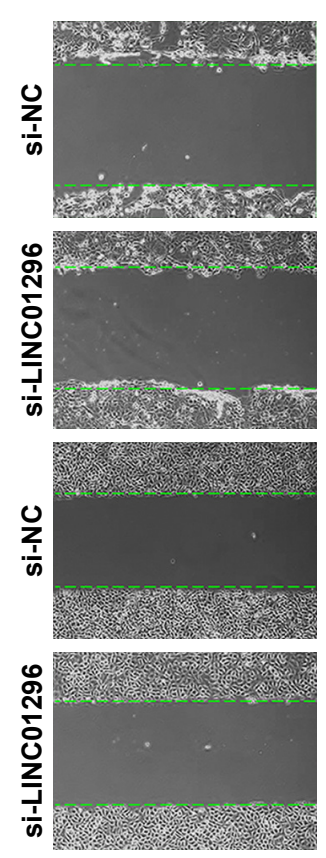

$\mathrm{Oh}$
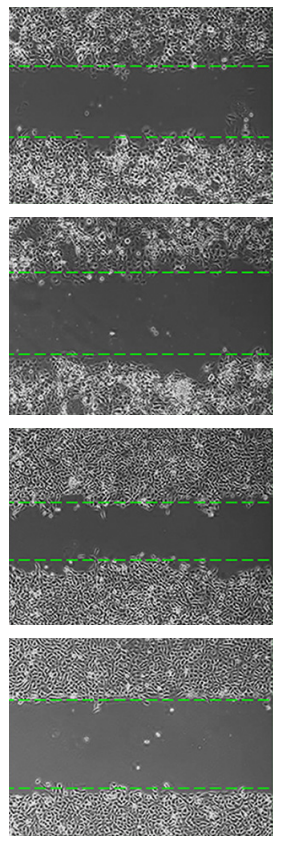

$18 \mathrm{~h}$
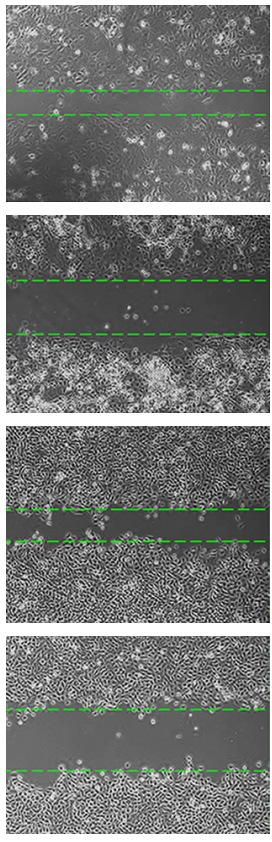

$24 \mathrm{~h}$
D
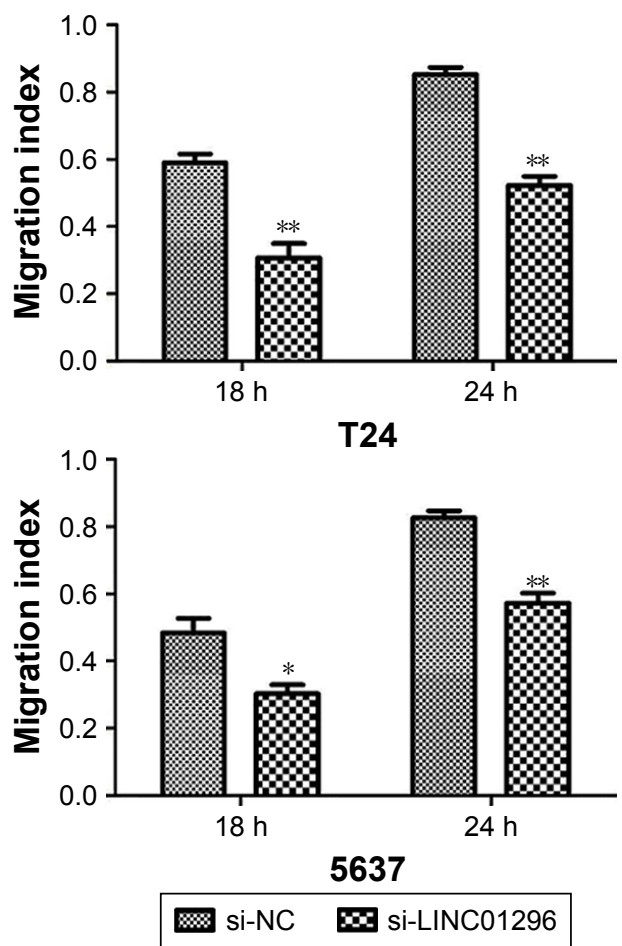

$\mathbf{F}$
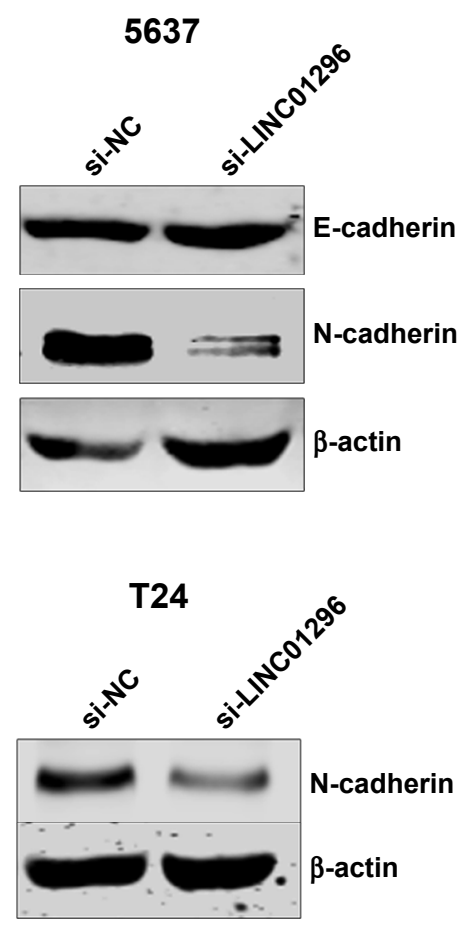

\section{si-NC}

Figure 4 LINCOI 296 knockdown suppressed the migration of bladder cancer cells.

Notes: (A, B) Transwell assays were used to measure the migration ability of T24 and 5637 cells transfected with si-LINC0I296 or si-NC. (C, D) Wound healing assays were used to measure the migration ability of T24 and 5637 cells transfected with si-LINC0I296 or si-NC. (E) qRT- PCR analyses were used to measure mRNA levels of $\mathrm{E}$-cadherin and N-cadherin following the treatment of bladder cells with anti-LINC0I296 siRNA. (F) Western blot assays were used to measure protein levels of E-cadherin and $\mathrm{N}$-cadherin following the treatment of bladder cells with anti-LINC0I 296 siRNA. Data are presented as mean $\pm S D$ of three independent experiments. $* P<0.05$, $* * \mathrm{P}<0.01$.

Abbreviations: NC, negative control; $\mathrm{qRT}-\mathrm{PCR}$, quantitative real-time polymerase chain reaction. 
functions, such as proliferation, invasion, and metastasis of cancer cells. ${ }^{24,25}$ For bladder cancer, numerous lncRNAs have been verified to take part in its carcinogenesis and development. Luo et $\mathrm{al}^{26}$ firstly found that the levels of H19 and inhibitors of DNA binding/differentiation 2 (ID2) are significantly increased in bladder cancer tissues, while the upregulation in H19 promotes the proliferation of bladder cancer cells by regulating ID2 expressions. As a lncRNA involved in bladder cancer metastasis, MALAT-1 is upregulated in bladder cancer tissues compared with that in adjacent normal tissues. Knockdown of MALAT-1 inhibits the in vitro invasion of bladder cancer cells by decreasing the expression of ZEB1, ZEB2 and Slug, which are shown to be associated with EMT, and by increasing the level of E-cadherin. ${ }^{27}$ Previous studies found that LINC01296 was upregulated in prostate cancer, gastric cancer, and cholangiocarcinoma. Upregulated LINC01296 was associated with a poor prognosis of patients with these types of cancer and could promote the proliferation and metastasis of cancer cells. ${ }^{10,28,29}$ Although LINC01296 has been studied in these types of cancer, the possible role of LINC01296 in bladder cancer remains to be clarified. In the present study, it was found that LINC01296 is upregulated in bladder cancer tissues, consistent with the results of GEPIA analysis and microarray assay conducted in the preliminary phase of this study. It was also indicated that the LINC01296 expression was significantly higher in patients with a higher clinical stage of bladder cancer, lymph node metastasis and a higher pathologic grade of the cancer. In addition, the results of Kaplan-Meier survival analysis showed that the high LINC01296 expression was associated with significantly worse OS. Furthermore, the results of univariate and multivariate analyses showed that LINC01296 acted as an independent risk factor in bladder cancer patients. A previous study also showed that the LINC01296 expression was significantly upregulated in bladder cancer cell lines compared with that in normal bladder cell lines, ${ }^{20}$ although the biomedical functions of LINC01296 in bladder cancer were not thoroughly studied in that study. This study focused on the function research of LINC01296 in vitro. First, the knockdown of LINC01296 significantly inhibited the proliferation and metastasis of T24 and 5637 cells, indicating that downregulated LINC01296 expressions could suppress the development of bladder cancer. The findings of this study also showed that LINC01296 may function as an oncogenic IncRNA in bladder cancer and potentially be considered as a novel biomarker and therapeutic target of this disease. Epithelial-to-mesenchymal transition plays an important role in the invasion and migration of various tumors, including bladder cancer. ${ }^{30-34}$ Recent research studies have indicated that lncRNA H19 plays a critical role in cancer metastasis by regulating EMT and mesenchymal-to-epithelial transition in bladder cancer. ${ }^{35}$ Furthermore, LINC01296 could take part in the metastasis of prostate cancer by regulating the EMT process. ${ }^{10}$ In the present study, it was also found that LINC01296 played an important role in the EMT process of bladder cancer, while the downregulation of LINC01296 could remarkably inhibit the EMT of T24 and 5637 cells, thus decreasing the expression of $\mathrm{N}$-cadherin and increasing the expression of E-cadherin. This study mechanistically affirmed that LINC01296 acts as a significant regulator of EMT in bladder cancer. However, lncRNAs could interact with DNA, RNA and protein to take part in the regulation of gene expressions and to exert biological functions. Nevertheless, the binding partner of LINC01296 and the consequences of LINC01296 upregulation were not investigated in this study. In addition, relevant signaling pathways responsible for the role of LINC01296 in the induction of EMT were not investigated. These issues should be studied in subsequent investigations.

\section{Conclusion}

In conclusion, this study showed that LINC01296 expression was significantly upregulated in bladder cancer tissues and cells compared with normal bladder tissues and cells. In addition, the LINC01296 expression was positively associated with clinical stages of bladder cancer, lymph node metastasis and pathologic grades of bladder cancer, indicating that the overexpression of LINC01296 may be used as a negative prognostic factor in bladder cancer patients. Knockdown of LINC01296 suppressed tumor progression through inhibiting the proliferation, growth and migration of cancer cells, indicating that LINC01296 played an important role in the development of bladder cancer. Furthermore, upregulated LINC01296 expression could promote bladder cancer metastasis through activating the EMT pathway. The results of this study can help to deepen the understanding of bladder cancer pathogenesis. In addition, it was shown that LINC01296 could be used as a molecular marker and a potential diagnostic/therapeutic target in the treatment of bladder cancer.

\section{Acknowledgment}

The study was supported by the Clinical Research Special Fund of Wu Jieping Medical Foundation (No. 320.6750.15227). Xiaofei Wang and Lei Wang are co-first authors for this paper.

\section{Disclosure}

The authors report no conflicts of interest in this work. 


\section{References}

1. Torre LA, Bray F, Siegel RL, Ferlay J, Lortet-Tieulent J, Jemal A. Global cancer statistics, 2012. CA Cancer J Clin. 2015;65(2):87-108.

2. Knowles MA, Hurst CD. Molecular biology of bladder cancer: new insights into pathogenesis and clinical diversity. Nat Rev Cancer. 2015;15(1):25-41.

3. Martin-Doyle W, Kwiatkowski DJ. Molecular biology of bladder cancer. Hematol Oncol Clin North Am. 2015;29(2):191-203.

4. Egerod FL, Bartels A, Fristrup N, et al. High frequency of tumor cells with nuclear Egr-1 protein expression in human bladder cancer is associated with disease progression. BMC Cancer. 2009;9:385.

5. Bo J, Yang G, Huo K, et al. microRNA-203 suppresses bladder cancer development by repressing bcl-w expression. FEBS J. 2011;278(5): 786-792.

6. Kim WJ, Kim EJ, Kim SK, et al. Predictive value of progressionrelated gene classifier in primary non-muscle invasive bladder cancer. Mol Cancer. 2010;9:3.

7. Xu SQ, Buraschi S, Morcavallo A, et al. A novel role for drebrin in regulating progranulin bioactivity in bladder cancer. Oncotarget. 2015;6(13):10825-10839.

8. Mahdavifar N, Ghoncheh M, Pakzad R, Momenimovahed Z, Salehiniya H. Epidemiology, incidence and mortality of bladder cancer and their relationship with the development index in the world. Asian Pac J Cancer Prev. 2016;17(1):381-386.

9. Qiu JJ, Yan JB. Long non-coding RNA LINC01296 is a potential prognostic biomarker in patients with colorectal cancer. Tumour Biol. 2015;36(9):7175-7183.

10. Wu J, Cheng G, Zhang C, et al. Long noncoding RNA LINC01296 is associated with poor prognosis in prostate cancer and promotes cancer-cell proliferation and metastasis. Onco Targets Ther. 2017;10: 1843-1852.

11. Monteleone NJ, Lutz CS. miR-708-5p: a microRNA with emerging roles in cancer. Oncotarget. 2017;8(41):71292-71316.

12. Xu YC, Liang CJ, Zhang DX, et al. LncSHRG promotes hepatocellular carcinoma progression by activating HES6. Oncotarget. 2017 ; 8(41):70630-70641.

13. Liu B, Ye B, Yang L, et al. Long noncoding RNA $\operatorname{lncKdm} 2 \mathrm{~b}$ is required for ILC3 maintenance by initiation of Zfp292 expression. Nat Immunol. 2017;18(5):499-508.

14. Svoboda P. Long and small noncoding RNAs during oocyte-to-embryo transition in mammals. Biochem Soc Trans. 2017;45(5):1117-1124.

15. Zhu P, Wang Y, Wu J, et al. LncBRM initiates YAP1 signalling activation to drive self-renewal of liver cancer stem cells. Nat Commun. 2016; 7:13608.

16. Faghihi F, Moustafa AA. Impaired neurogenesis of the dentate gyrus is associated with pattern separation deficits: a computational study. J Integr Neurosci. 2016;15(3):277-293.

17. Kornienko AE, Guenzl PM, Barlow DP, Pauler FM. Gene regulation by the act of long non-coding RNA transcription. BMC Biol. 2013;11:59.

18. Lee JT, Bartolomei MS. X-inactivation, imprinting, and long noncoding RNAs in health and disease. Cell. 2013;152(6):1308-1323.
19. Tang Z, Li C, Kang B, Gao G, Li C, Zhang Z. GEPIA: a web server for cancer and normal gene expression profiling and interactive analyses. Nucleic Acids Res. 2017;45(W1):W98-W102.

20. Qin YC, Wang L, Gong YB. Long non-coding RNA DUXAP9, DUXAP10 and LINC01296 are highly expressed in urothelial carcinoma of the bladder. J Clin Urol. 2016;31(10):921-925.

21. Pan J, Li X, Wu W, et al. Long non-coding RNA UCA1 promotes cisplatin/gemcitabine resistance through CREB modulating miR196a-5p in bladder cancer cells. Cancer Lett. 2016;382(1):64-76.

22. Luo M, Li Z, Wang W, Zeng Y, Liu Z, Qiu J. Long non-coding RNA H19 increases bladder cancer metastasis by associating with EZH2 and inhibiting E-cadherin expression. Cancer Lett. 2013;333(2):213-221.

23. Rausch S, Hennenlotter J, Todenhöfer T, et al. Impaired estimated glomerular filtration rate is a significant predictor for non-muscle-invasive bladder cancer recurrence and progression - introducing a novel prognostic model for bladder cancer recurrence. Urol Oncol. 2014;32(8): 1178-1183.

24. Gutschner T, Diederichs S. The hallmarks of cancer: a long non-coding RNA point of view. RNA Biol. 2012;9(6):703-719.

25. Esteller M. Non-coding RNAs in human disease. Nat Rev Genet. 2011; 12(12):861-874.

26. Luo M, Li Z, Wang W, Zeng Y, Liu Z, Qiu J. Upregulated H19 contributes to bladder cancer cell proliferation by regulating ID2 expression. FEBS J. 2013;280(7):1709-1716.

27. Ying L, Chen Q, Wang Y, Zhou Z, Huang Y, Qiu F. Upregulated MALAT-1 contributes to bladder cancer cell migration by inducing epithelial-to-mesenchymal transition. Mol Biosyst. 2012;8(9): 2289-2294.

28. Qin QH, Yin ZQ, Li Y, Wang BG, Zhang MF. Long intergenic noncoding RNA 01296 aggravates gastric cancer cells progress through miR-122/MMP-9. Biomed Pharmacother. 2018;97:450-457.

29. Zhang D, Li H, Xie J, et al. Long noncoding RNA LINC01296 promotes tumor growth and progression by sponging miR-5095 in human cholangiocarcinoma. Int J Oncol. 2018;52(6):1777-1786.

30. Zheng ZG, Xu H, Suo SS, et al. The essential role of H19 contributing to cisplatin resistance by regulating glutathione metabolism in highgrade serous ovarian cancer. Sci Rep. 2016;6:26093.

31. Huang C, Cao L, Qiu L, et al. Upregulation of H19 promotes invasion and induces epithelial-to-mesenchymal transition in esophageal cancer. Oncol Lett. 2015;10(1):291-296.

32. Matouk IJ, Halle D, Raveh E, Gilon M, Sorin V, Hochberg A. The role of the oncofetal H19 lncRNA in tumor metastasis: orchestrating the EMT-MET decision. Oncotarget. 2016;7(4):3748-3765.

33. Yang G, Lu W, Yu D, et al. Quantitative analysis of differential proteome expression in epithelial-to-mesenchymal transition of bladder epithelial cells using SILAC method. Molecules. 2016;21(1):84.

34. Yun SJ, Kim WJ. Role of the epithelial-mesenchymal transition in bladder cancer: from prognosis to therapeutic target. Korean J Urol. 2013;54(10):645-650.

35. Lv M, Zhong Z, Huang M, Tian Q, Jiang R, Chen J. lncRNA H19 regulates epithelial-mesenchymal transition and metastasis of bladder cancer by miR-29b-3p as competing endogenous RNA. Biochim Biophys Acta Mol Cell Res. 2017;1864(10):1887-1899.
OncoTargets and Therapy

\section{Publish your work in this journal}

OncoTargets and Therapy is an international, peer-reviewed, open access journal focusing on the pathological basis of all cancers, potential targets for therapy and treatment protocols employed to improve the management of cancer patients. The journal also focuses on the impact of management programs and new therapeutic agents and protocols on

Submit your manuscript here: http://www.dovepress.com/oncotargets-and-therapy-journal
Dovepress

patient perspectives such as quality of life, adherence and satisfaction. The manuscript management system is completely online and includes a very quick and fair peer-review system, which is all easy to use. Visit http://www.dovepress.com/testimonials.php to read real quotes from published authors. 